\title{
Ring strain and total syntheses of modified macrocycles of the isoplagiochin type
}

\author{
Andreas Speicher ${ }^{*}$, Timo Backes, Kerstin Hesidens and Jürgen Kolz
}

\section{Full Research Paper}

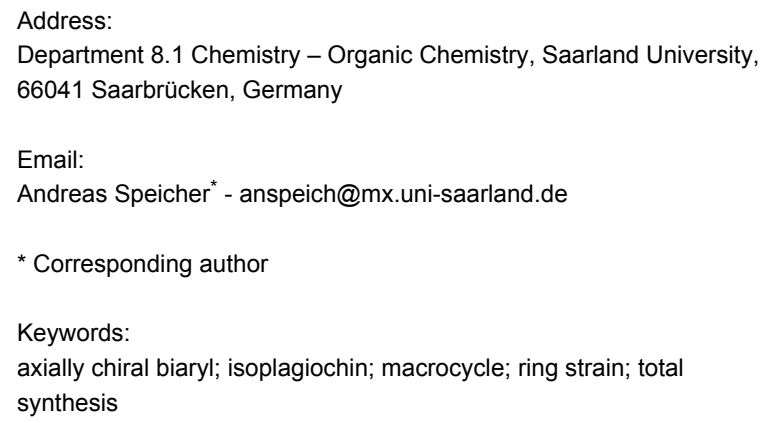

\begin{abstract}
Macrocycles of the bisbibenzyl-type are natural products that are found exclusively in bryophytes (liverworts). The molecular framework of the subtype "isoplagiochin" is of substantial structural interest because of the chirality of the entire molecule, which arises from two biaryl axes in combination with two helical two-carbon units in a cyclic arrangement. From a structural as well as a synthetic point of view we report on the total synthesis of compounds which possess more rigid two-carbon biaryl bridges like stilbene $(E$ or $Z$ ) or even tolane moieties which were introduced starting with a Sonogashira protocol. The McMurry method proved to be a powerful tool for the cyclization to these considerably ring-strained macrocycles.
\end{abstract}

\section{Introduction}

The cyclic bisbibenzyls isoplagiochin C (1) and D (2) were isolated from the liverworts Plagiochila fruticosa [1], Plagiochila deflexa [2], Herbertus sakuraii [3] and Lepidozia fauriana [4] (Figure 1). Recently, an increasing number of different biological activities of phenolic compounds of the bisbibenzyl type were reported [5-12].

Furthermore, the isoplagiochin framework proved to be of substantial structural interest because of the chirality of the entire molecule. The existence of atropoisomers [3] prompted us to perform detailed studies on the chirality of $\mathbf{1}$ and $\mathbf{2}$ including HPLC-CD experiments. Assuming the existence of

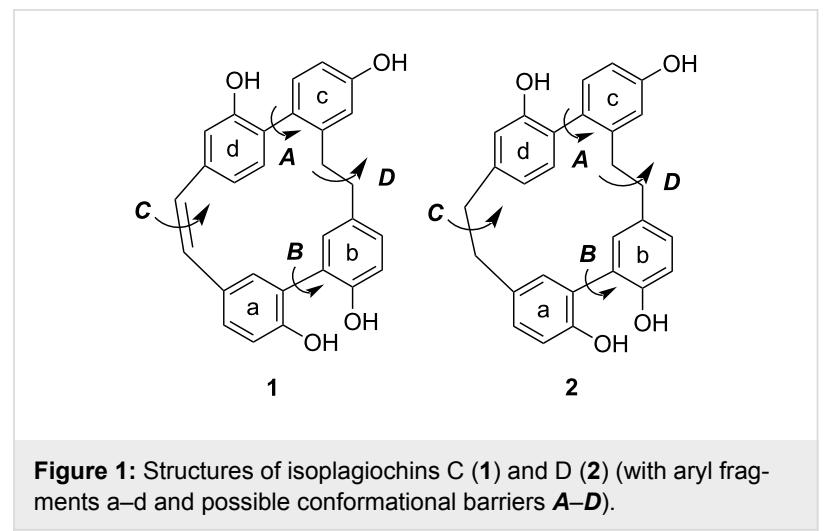


three formal stereogenic elements $\boldsymbol{A}-\boldsymbol{C}$ (Figure 1), a total of up to $2^{3}=8$ conformers (four diastereomers with their enantiomers) in principle are possible for $\mathbf{1}$ (and analogously for $\mathbf{2}$ ) (Figure 2). The ethylene bridge $\boldsymbol{D}$ is more flexible in these two molecules. Temperature dependent NMR investigations did not provide any evidence for the existence of different diastereomers configurationally stable within the NMR timescale. AM1based conformational analysis and MD investigations clearly confirmed the biaryl axis $\boldsymbol{A}$ to be configurationally stable at room temperature due to the second (more flexible) biaryl axis $\boldsymbol{B}$, an (even more flexible) helical stilbene unit $\boldsymbol{C}$ and their combination with the ring-strain of the entire molecule.

By experimental and quantum chemical CD (circular dichroism) investigations, the absolute configuration of the first natural compound of this type, isoplagiochin $\mathrm{C}$ from $P$. deflexa, was established as $\left(P_{A}\right) \mathbf{- 1}$ and the energy of racemization was calculated and measured to be $102 \mathrm{~kJ} / \mathrm{mol}$, approximately [13]. The enantiomers $\left(P_{A}\right) \mathbf{- 1}$ and $\left(M_{A}\right) \mathbf{- 1}$ are each manifested in the four imaginable, rapidly interconverting diastereomers whereby the $\mathrm{C} 2$ conformations were estimated as the global minima (Figure 2). Similar experiments and calculations were performed for isoplagiochin D (2) and for some chlorinated derivatives. The stereochemical correlation between isoplagiochin C (1) and isoplagiochin D (2) was also confirmed experimentally by hydrogenolysis of enantiopure samples of $\left(P_{A}\right)$-1 to give and $\left(P_{A}\right)-2$ (Scheme 1), and of $\left(M_{A}\right)-1$ to give and $\left(M_{A}\right)-\mathbf{2}$, respectively, without any racemization [14].

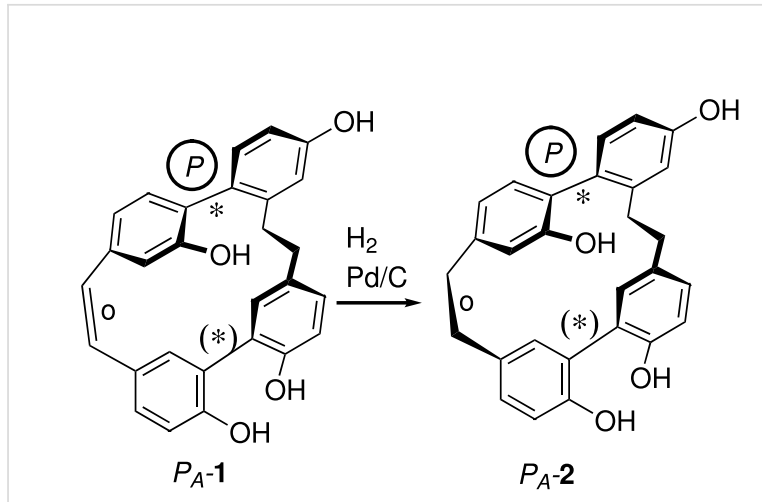

Scheme 1: Stereochemical correlation for 1 and 2. (*: configurationally stable, $\left({ }^{\star}\right)$ : configurationally semi-stable; o: configurationally unstable).

The natural compounds isoplagiochin D (2) and C (1) possess two saturated ethylene bridges (between rings $\mathbf{a}-\mathbf{d}$ and $\mathbf{b}-\mathbf{c}$ ) or just one cis-configurated double bond ('stilbene bridge') between rings a-d. From a structural as well as a synthetic point of view we were interested in compounds which possess various unsaturated stilbene ( $E$ or $Z$ ) or even tolane bridges between the two biaryl units $\mathbf{a}-\mathbf{b}$ and $\mathbf{c}-\mathbf{d}$. These structures should be more rigid with respect to the ring strain enhanced by geometrically fixed two-carbon bridges. But for ring strain considerations, the real cyclization product possessing at least additional phenolic protecting groups should be studied.

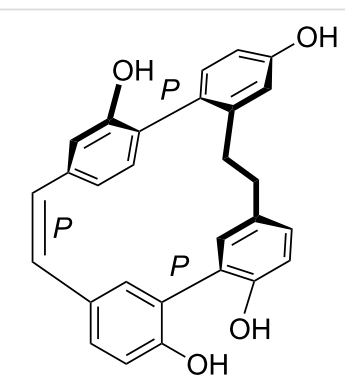

$P_{A}-\mathbf{1}_{\mathrm{C} 1}$

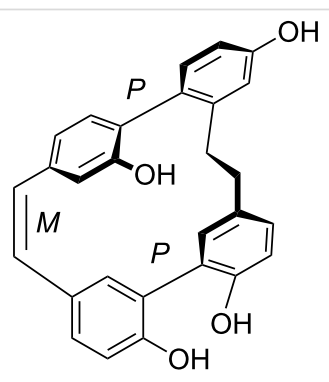

$P_{A}-1_{\mathrm{C} 2}$

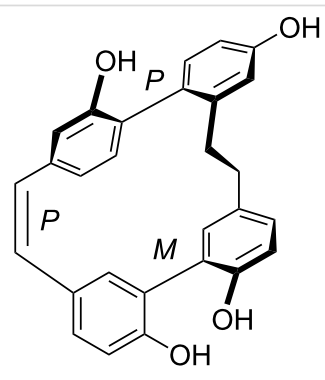

$P_{A}-1_{C 3}$

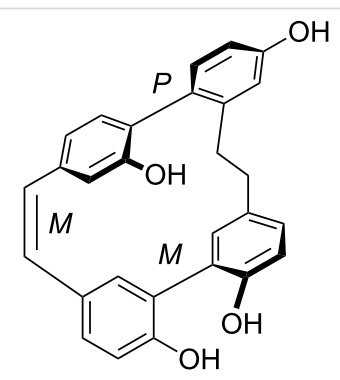

$P_{A}-1_{\mathrm{C} 4}$

mirror plane
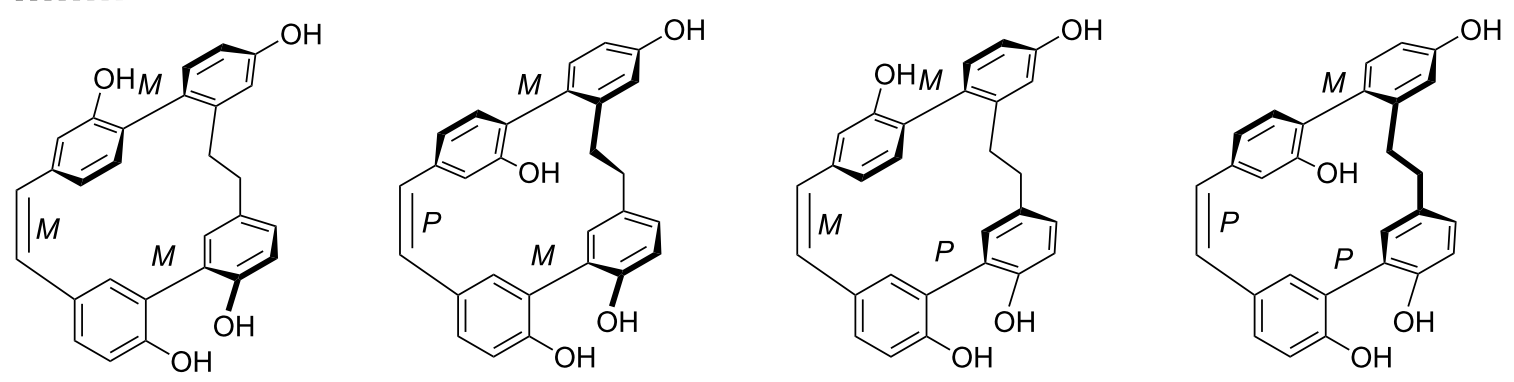

$M_{A}-1_{C 1}$

$M_{A}-1_{\mathrm{C} 2}$

$M_{A}-1_{C 3}$

$M_{A}-1_{C 4}$

Figure 2: Possible stereoisomers of 1 as conformers C1-C4 relative to the configurationally stable biaryl axis $\boldsymbol{A}$ with the stereo descriptors $P$ or $M$. 


\section{Results and Discussion}

In our total syntheses of the racemic natural compounds $\mathbf{1}$ and $\mathbf{2}$ reported previously [15] the common and crucial precursor was the tetramethyl ether 3. Surprisingly and in contrast to the NMR results mentioned above for $\mathbf{1}$, closer NMR examination now revealed two sets of signals for $\mathbf{3}$ indicating two conformers (ratio $1.3: 1$ ) stable within the NMR time scale. This can be interpreted as due to a much less flexible axis $\boldsymbol{B}$ resulting from the stereochemically more demanding methoxy groups in the $o, o^{\prime}$-positions (Figure 3).
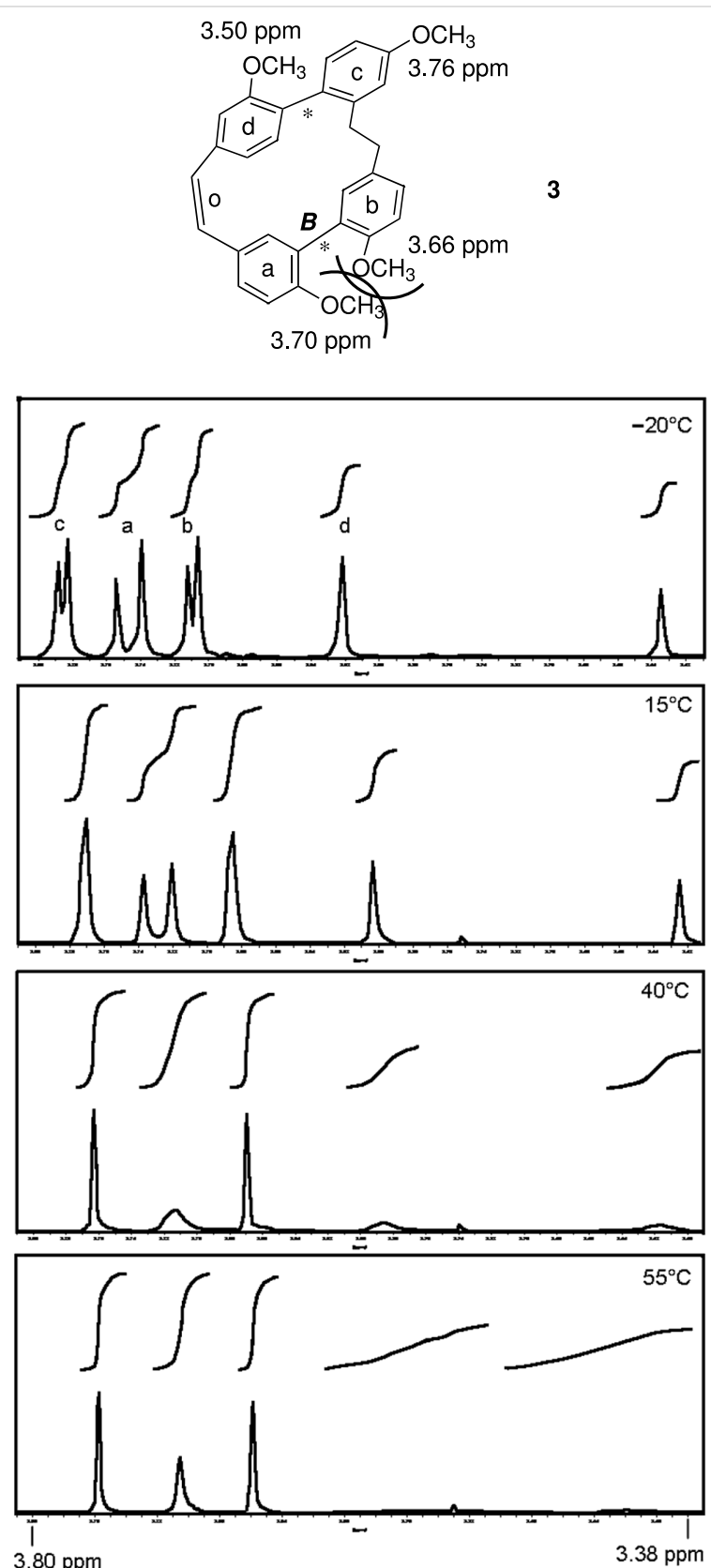

Figure 3: Temperature dependent ${ }^{1} \mathrm{H}$ NMR and assignment of methoxy signals in the tetramethyl ether 3 .
Indeed, temperature dependent ${ }^{1} \mathrm{H}$ NMR investigations from -20 up to $55^{\circ} \mathrm{C}$ resulted in coalescence of the methoxy signals. By an approximation method [16] as well as by exact line form analysis [17] the rotation barrier could be calculated to $\sim 66 \mathrm{~kJ} /$ mol indicating that the conformers are stable within the NMR time scale but cannot be separated. By 2D NMR experiments (COSY, HSQC, HMBC) at $100{ }^{\circ} \mathrm{C}$ four sharp signals could be assigned to the methoxy groups in rings a-d (Figure 3).

To gain insight into possible ring strain limitations for the preparation and stability of modified macrocycles of the isoplagiochin type we performed molecular mechanic and semiempirical calculations for tetramethyl protected target compounds. Together with the molecules "in hand" they clearly demonstrated that the bridge between a-d should be saturated or at least a $Z$ double bond as in the natural compounds $\mathbf{1}$ (cf. 3) and 2 (cf. 4). An $E$ double bond or an alkyne moiety are not well tolerated (see e.g. 8, 9), even in the system with the lowest possible strain, a saturated $\mathbf{b}-\mathbf{c}$-bridge. This can be deduced mainly from the substantial angular strain in the alkyne moiety (see 8) or from the distortion in the (E)-alkene moiety (see 9) as well as in torsion stress looking at the aromatic rings $\mathbf{a}$ and $\mathbf{d}$ in both candidates. The obvious reason for these effects is the para-insertion of the aryl moiety $\mathbf{d}$ into the macrocyclic system. In contrast, for the bridge $\mathbf{b}-\mathbf{c}$, the $Z$ and $E$ double bond as well as a tolane bridge should be more easily realizable with respect to angular strain and torsion stress (see compounds 5-7 in Table 1).

As a consequence of these results we were interested in the syntheses of the new bisbibenzyl frameworks 5-7. Our previously reported total syntheses of the racemic natural compounds $\mathbf{1}$ and $\mathbf{2}$ [15,19] or some derivatives [20] could not be adopted because they do not give rise to specific double bond geometry or even an alkyne moiety between rings $\mathbf{b}$ and $\mathbf{c}$.

Our new strategy of synthesis involved a consecutive coupling of the rings a-b-c-d whereby the two-carbon bridge $\mathbf{b}-\mathbf{c}$ would be introduced by a Sonogashira type reaction yielding an alkyne moiety (see 7), from which a $Z$ double bond (for 5) or an $E$ double bond may be obtainable by Lindlar-type hydrogenation and (for 6) by subsequent $Z \rightarrow E$ isomerization (Scheme 2). The final ring closure using a McMurry protocol (probably more rigorous for ring-strained frameworks than the Wittig-protocol in the syntheses of $\mathbf{3} / \mathbf{4}$ ) should give rise only to the $Z$ geometry at the a-d stilbene bridge.

The $\mathbf{a}-\mathbf{b}$ part 14 [15] of the target molecules was prepared by Suzuki coupling of the boronic acid $\mathbf{1 2}$ with its precursor 11, readily available from 3-bromo-4-methoxybenzaldehyde (10) [21] by an improved procedure [22]. The aldehyde $\mathbf{1 3}$ was 
Table 1: AM1 energies [18], angular strain and torsion stress of different modified macrocycles of the isoplagiochin type.

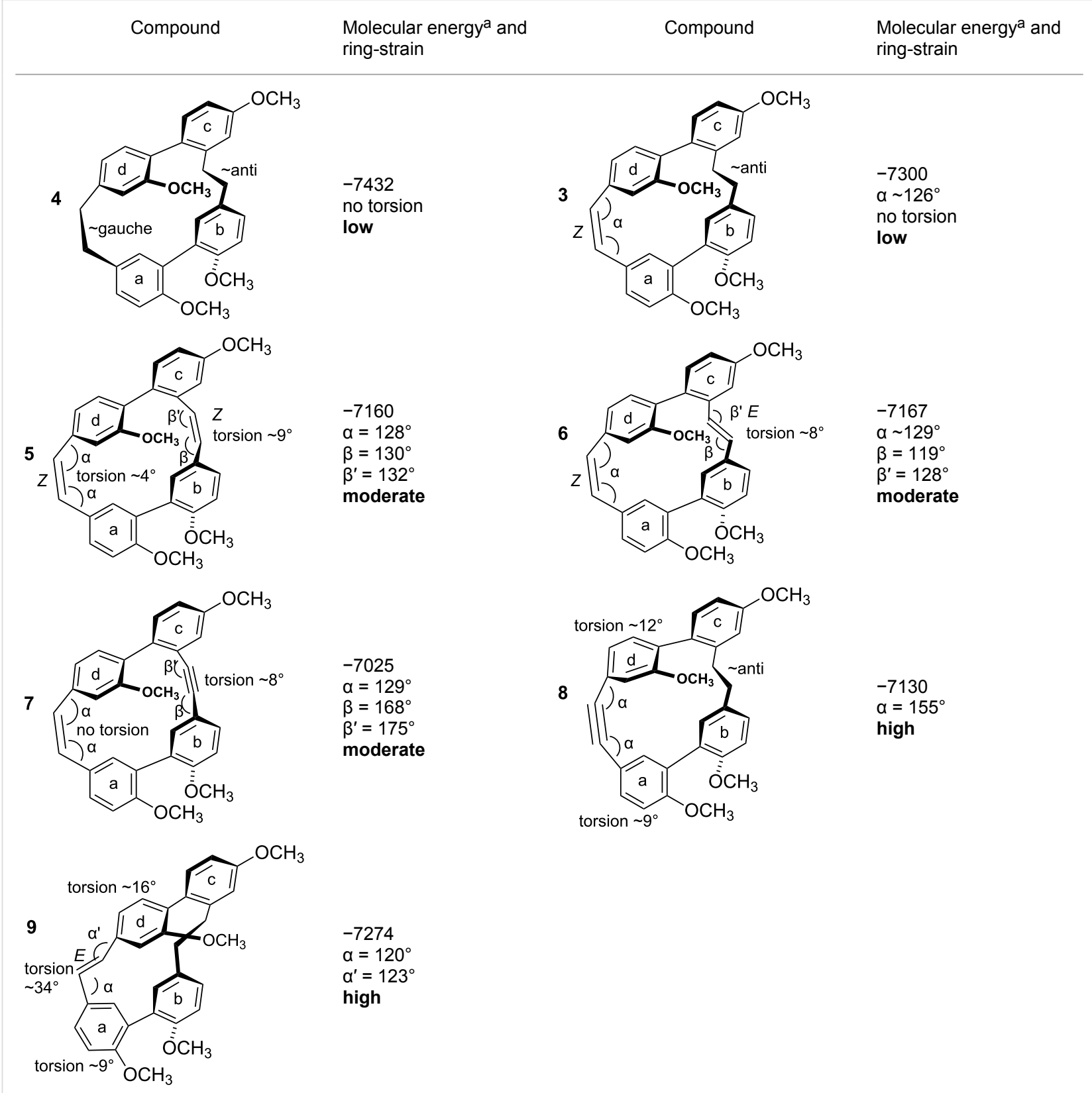

aThe calculations are given for the conformations resembling the most stable $P_{A}{ }^{-1}{ }_{\mathrm{C} 2}$ conformer (see Figure 1). No significant deviations were observed for other conformers. Note that molecular energy values should only be compared for isomeric compounds.

transformed into the terminal alkyne $\mathbf{1 4}$ by chain extension [23] (Scheme 3).

The coupling of the terminal alkyne $\mathbf{1 4}$ with the iodoarene $\mathbf{1 5}$ [24] as the "c" building block using a typical Sonogashira protocol $\left[\mathrm{Pd}\left(\mathrm{PPh}_{3}\right)_{4}, \mathrm{CuI}, \mathrm{Et}_{3} \mathrm{~N}\right]$ gave - even under an argon-hydrogen atmosphere [25] - only $30 \%$ yield beside the undesired homo-coupling product 17. A modified and palladium free procedure, however [26], resulted - after acidic workup liberating the aldehyde group - in a satisfactory yield of the mixed tolane $\mathbf{1 6}$ as the a-b-c precursor (Scheme 4).

Two different building blocks $\mathbf{2 2}$ and $\mathbf{2 6}$ were prepared as the "d" ring for Suzuki coupling with the bromoarene $\mathbf{1 6}$ (Scheme 5). First, iodination of 3-hydroxybenzoic acid (18) followed by double methylation and saponification of the methyl ester yielded 4-iodo-3-methoxybenzoic acid (19) which could be reduced in two steps [27] to the benzylic alcohol 20 

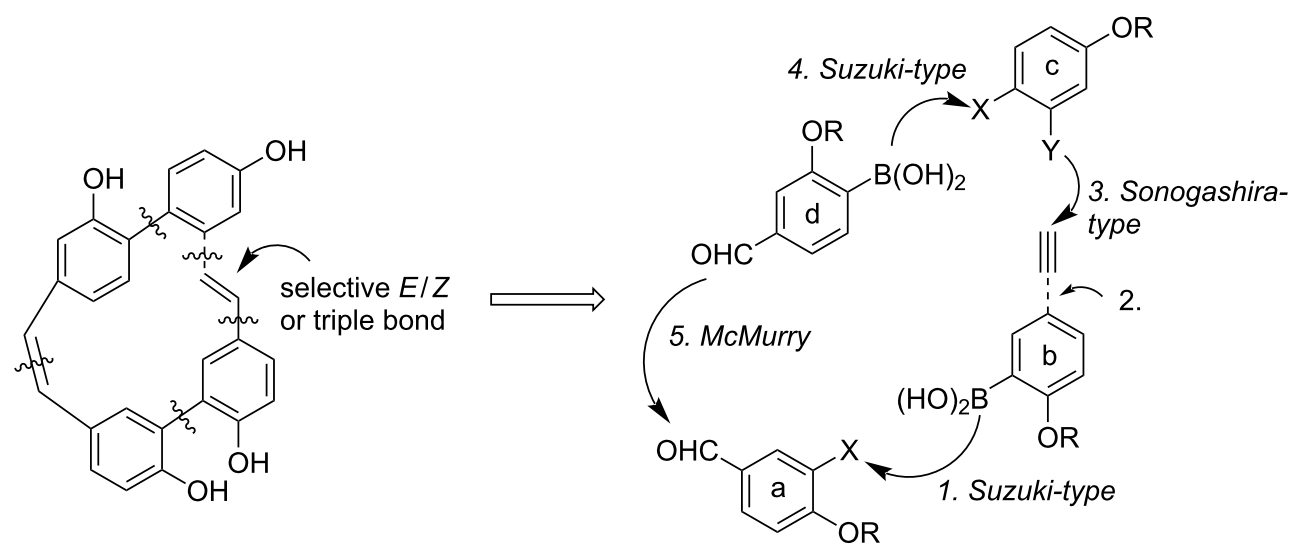

Scheme 2: Strategy of synthesis for the macrocycles 5-7.

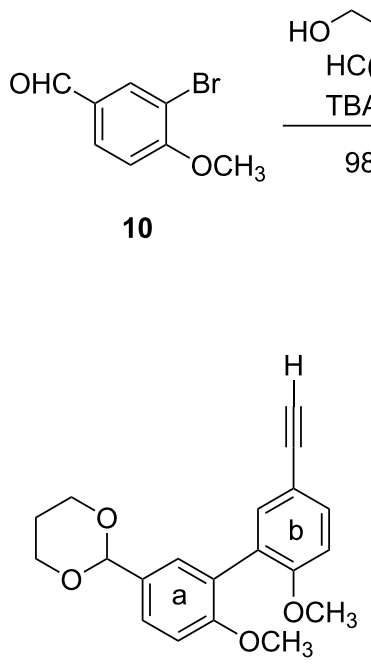

14

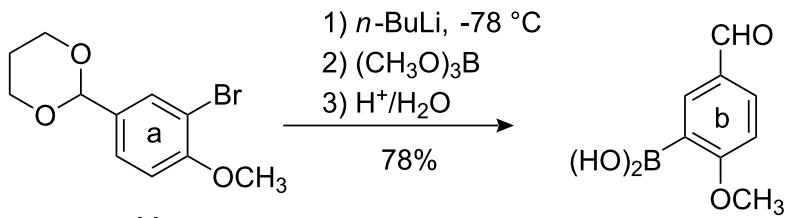

11

12

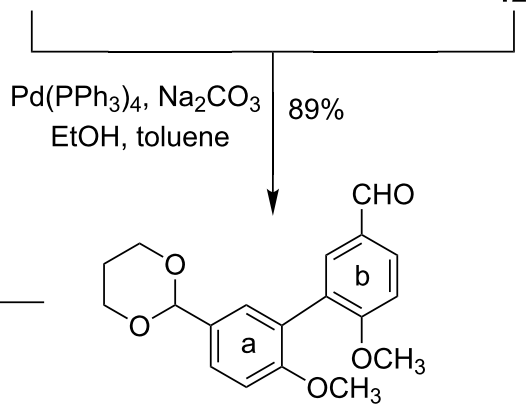

13

Scheme 3: Preparation of the terminal alkyne 13 as a-b part (TBATB = tetrabutylammonium tribromide).

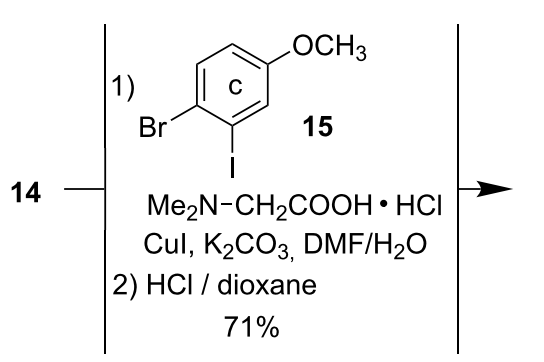

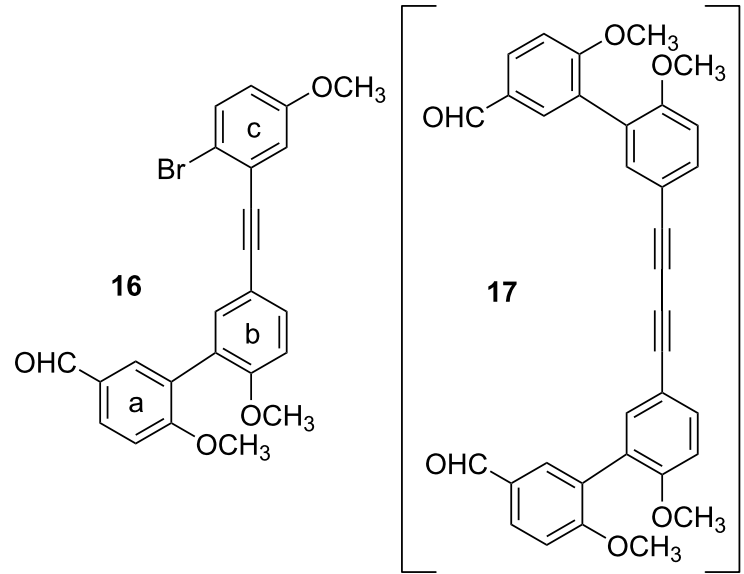

Scheme 4: Sonogashira-type coupling to the tolane 16. 
[28]. Protection as the THP acetal 21 was essential before preparing the boronic acid 22. Previously (see [15]), we reported on a synthesis of $\mathbf{2 2}$ starting with the bromination of 3-methoxybenzyl alcohol to 4-bromo-3-methoxybenzyl alcohol. In contrast to the original contribution [29] bromination lead to the formation of 2-bromo-5-methoxybenzyl alcohol, exclusively.

Second, the triflate $\mathbf{2 4}$ was obtained from vanillin (23) and was further dioxane-protected at the aldehyde function (to 25) and directly transformed into the arylboronate $\mathbf{2 6}$ using pinacolborane [30].

Suzuki coupling of the boronic acid $\mathbf{2 2}$ with the bromoarene $\mathbf{1 6}$ under carefully optimized conditions followed by acidic workup afforded the hydroxyaldehyde $\mathbf{2 7}$ as precursor for cyclization (Scheme 6). A cyclization protocol for the intramolecular Wittig reaction, well established $[15,20]$ for the synthesis of the tetramethyl ether of isoplagiochin C (1), however failed in this case. This might be due to the enhanced ring strain. Since the McMurry reaction would provide more rigorous conditions for the formation of ring-strained frameworks, the hydroxyaldehyde $\mathbf{2 7}$ was oxidized to the dialdehyde $\mathbf{2 8}$. It is worth mentioning that $\mathbf{2 8}$ could be obtained more straightforwardly and in higher overall yield directly from the bromoarene 16 and the arylboronate $\mathbf{2 6}$.

Indeed, the cyclization of $\mathbf{2 8}$ to the macrocycle $\mathbf{7}$ bearing an "alkyne bridge" was successful in moderate yield using a McMurry protocol (Scheme 7) [32]. Hydrogenation of the tolane 28 using Lindlar conditions selectively afforded the $(Z)$ alkene 29 from which the $(E)$-alkene $\mathbf{3 0}$ could be obtained selectively as well by an isomerization method [33,34]. From both acyclic precursors 29 and $\mathbf{3 0}$ the macrocycles 5 and $\mathbf{6}$ could be obtained analogously to 7 . In all cases and as expected, the new stilbene bridge was formed in the $Z$ geometry exclusively. For the new compounds 5-7 conformational studies by measuring temperature dependent NMR spectra clearly indicated significant rotational barriers of about $70 \mathrm{~kJ} / \mathrm{mol}$ for the biaryl axis $\boldsymbol{B}$ (see Supporting Information for each compound). These values, however, in comparison with the calculation for compound 3 (see Figure 3) indicate that the conformational flexibility of the macrocycles now synthesized is not significantly diminished with respect to this second biaryl axes. This result is also in accordance with the only moderately enhanced ring strain predicted for 5-7 compared to the parent compounds 3/4 (refer to Table 1).<smiles>COc1cc(C(=O)O)ccc1I</smiles><smiles>COc1cc(COP)ccc1OCCOP</smiles>

1) $n-\mathrm{BuLi}$

2) $\mathrm{B}\left(\mathrm{OMe}_{3}\right)$

3) $\mathrm{H}_{2} \mathrm{O}$ $61 \%$<smiles>COc1cc(COP)ccc1I</smiles>

21<smiles>CCCOc1ccc(C2OCCCO2)cc1OC</smiles><smiles>CC1(C)OBOC1(C)C</smiles>

$\mathrm{PdCl}_{2}(\mathrm{dppf})^{\mathrm{d}}$ $\mathrm{Et}_{3} \mathrm{~N}$, dioxane $55 \%$

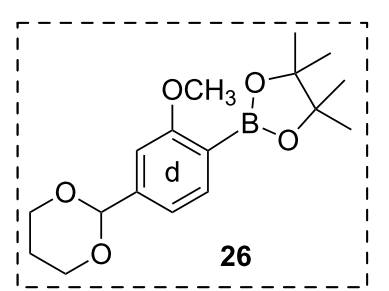<smiles>COc1cc(CO)ccc1I</smiles>

20

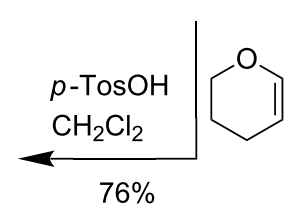

$76 \%$<smiles>[R]Oc1ccc(C=O)cc1OC</smiles><smiles>CCOC(Br)(Br)C(C)(Br)Br</smiles>

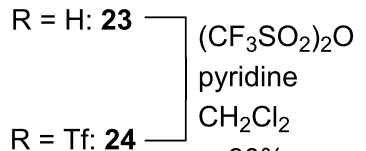

90\%

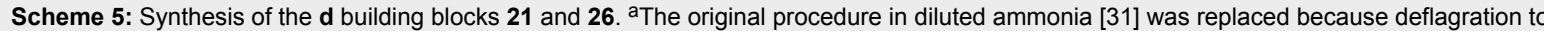
detonation was observed in some cases due to formation of nitrogen iodide $\left(\mathrm{NI}_{3}\right)$. ${ }^{\mathrm{b}}$ Attempts to direct reduction of the methyl ester or the carboxylic acid using e. g. $\mathrm{LiAlH}_{4}$ failed due to concomitant deiodination. ${ }^{\mathrm{C}}$ Protection of the aldehyde was obligatory to prevent exclusive pinacolborane reduction. ${ }^{d} d p p f: 1,1^{\prime}$-bis(diphenylphosphino)ferrocene. 


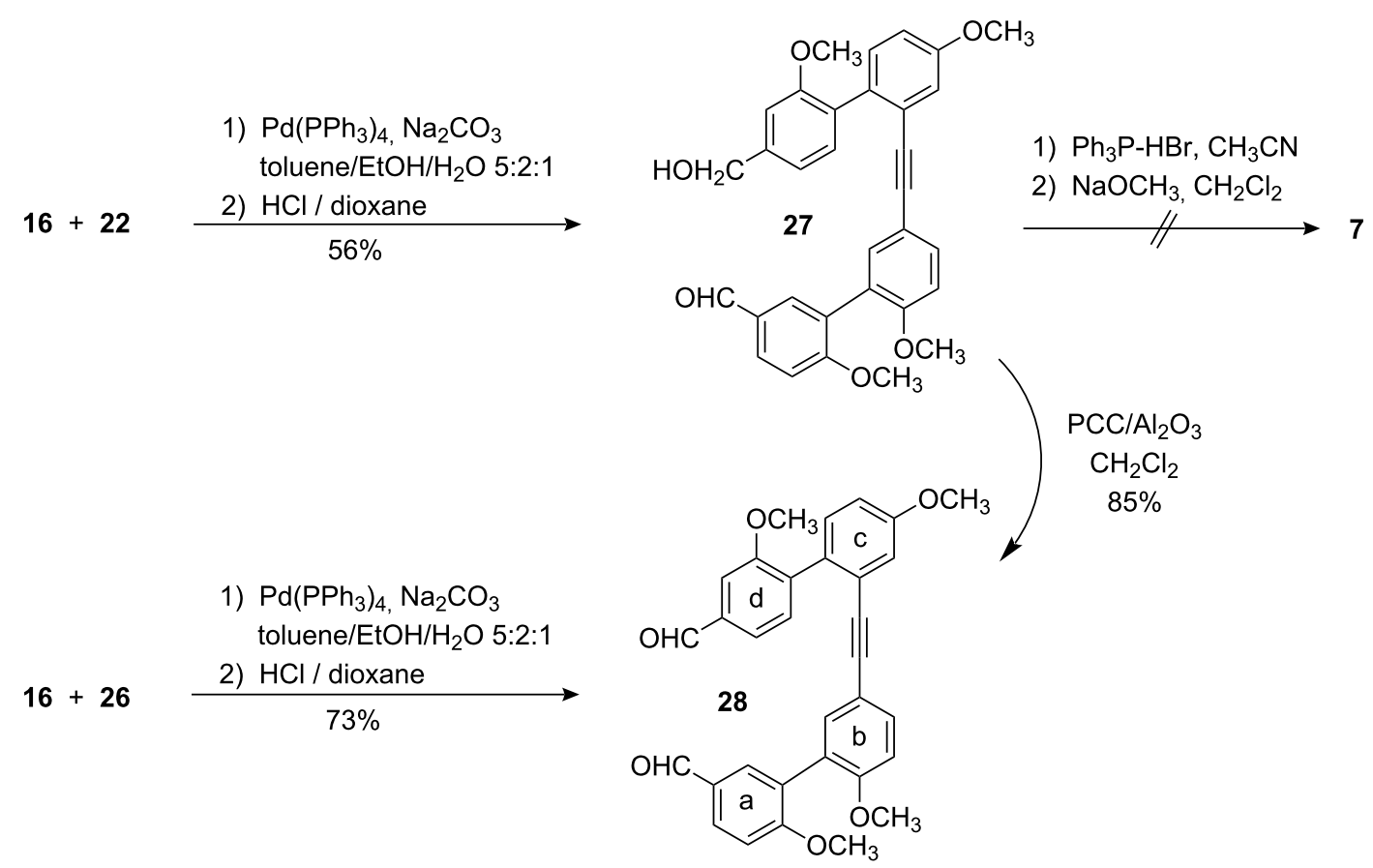

Scheme 6: Synthesis of the tolane precursors $\mathbf{2 7}$ and $\mathbf{2 8}$ for cyclization.

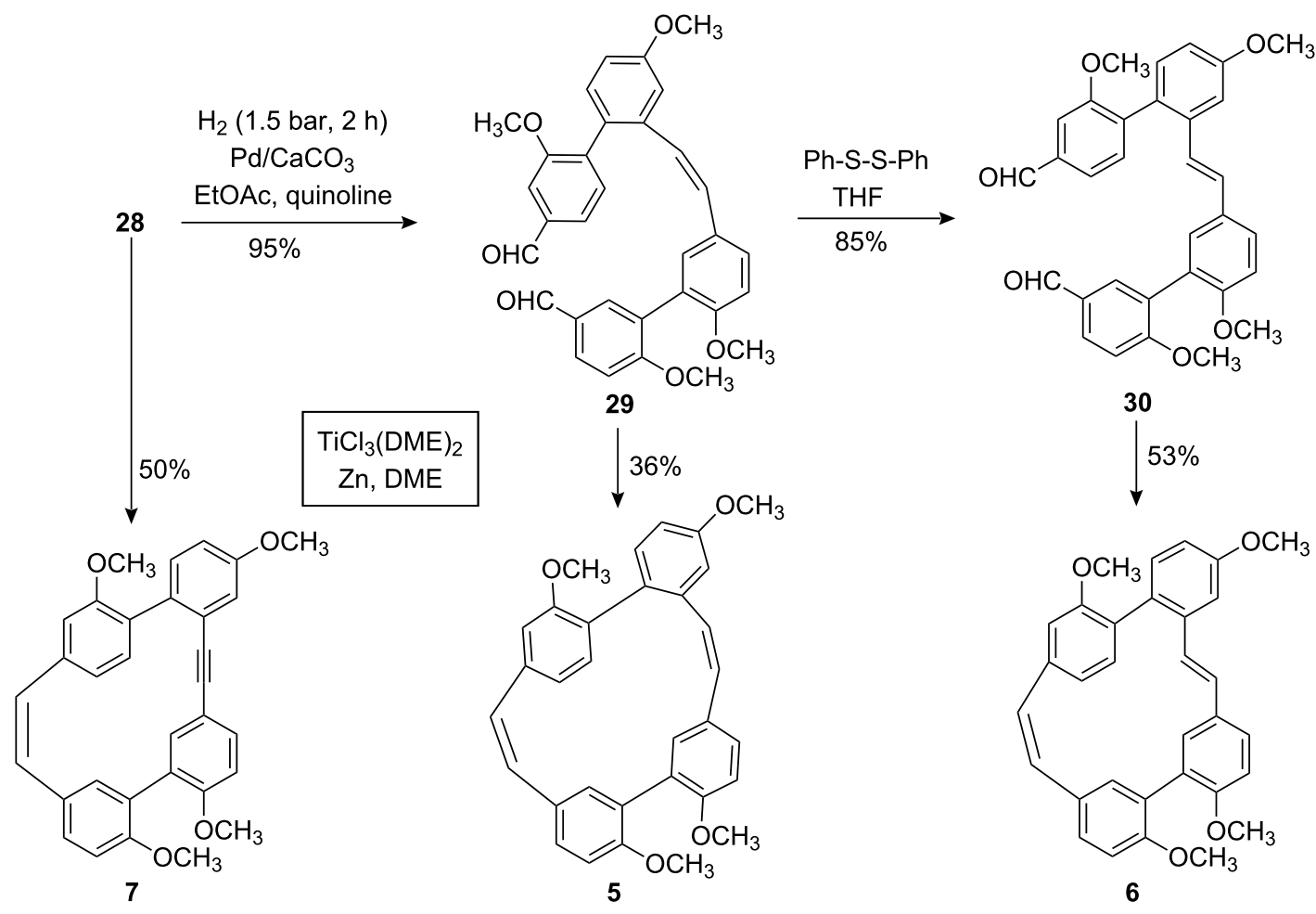

Scheme 7: Synthesis of the modified macrocycles 5-7 from the dialdehyde precursors 28-30. 


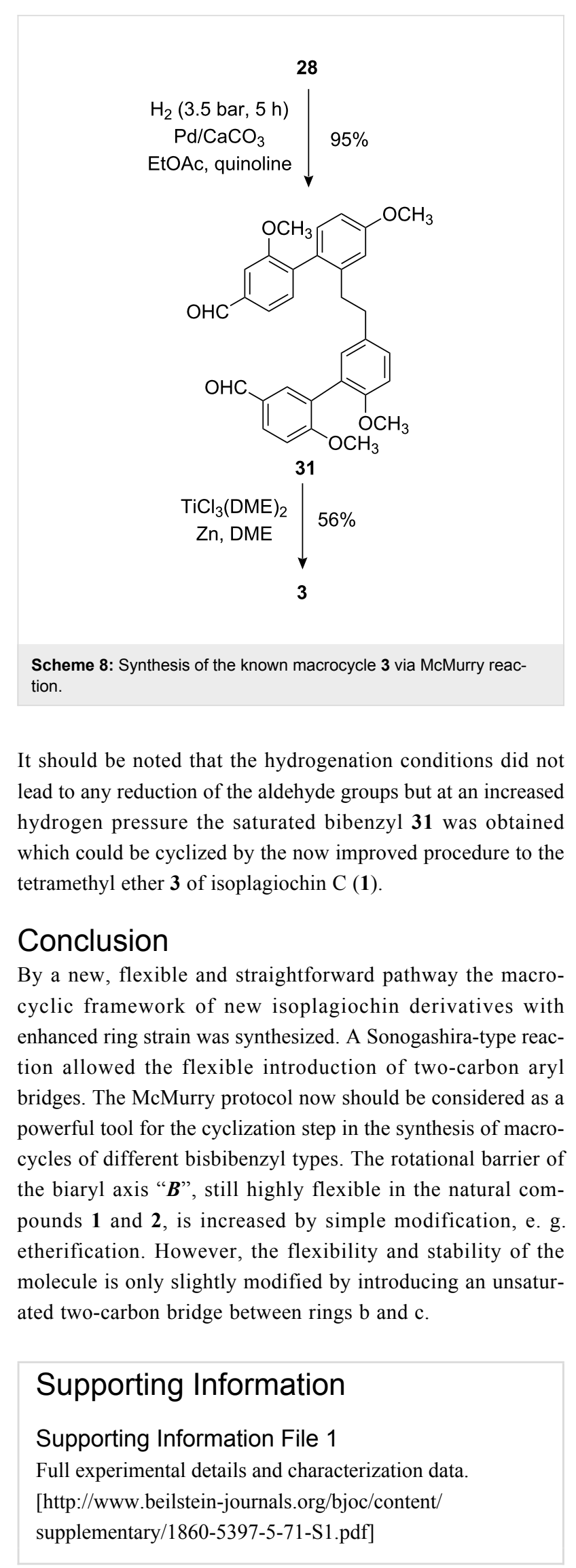

\section{References}

1. Hashimoto, T.; Kanayama, S.; Kan, Y.; Tori, M.; Asakawa, Y. Chem. Lett. 1996, 741-742. doi:10.1246/cl.1996.741

2. Anton, H.; Schoeneborn, R.; Mues, R. Phytochemistry 1999, 52, 1639-1645. doi:10.1016/S0031-9422(99)00394-5

3. Hashimoto, T.; Irita, H.; Takaoka, S.; Tanaka, M.; Asakawa, Y. Tetrahedron 2000, 56, 3153-3159. doi:10.1016/S0040-4020(00)00236-2

4. Shy, H.-S.; Wu, C.-L. J. Asian Nat. Prod. Res. 2006, 8, 723-731. doi:10.1080/10286020500247392

5. Harinantenaina, L.; Quang, D. N.; Takeshi, N.; Hashimoto, T.; Kohchi, C.; Soma, G.-I.; Asakawa, Y. J. Nat. Prod. 2005, 68, 1779-1781. doi:10.1021/np0502589

6. Guo, X.-L.; Leng, P.; Yang, Y.; Yu, L.-G.; Lou, H.-X. J. Appl. Microbiol. 2008, 104, 831-838. doi:10.1111/j.1365-2672.2007.03617.x

7. Leng, P.; Guo, X.-L.; Yang, Y.; Lou, H. X. Chin. Pharm. J. (Beijing) 2007, 42, 349-352.

8. Niu, C.; Qu, J.-B.; Lou, H.-X. Chem. Biodiversity 2006, 3, 34-40. doi:10.1002/cbdv.200690004

9. Qu, J.; Xie, C.; Guo, H.; Yu, W.; Lou, H. Phytochemistry 2007, 68, 1767-1774. doi:10.1016/j.phytochem.2007.04.036

10. Shi, Y.-Q.; Liao, Y.-X.; Qu, X.-J.; Yuan, H.-Q.; Li, S.; Qu, J.-B.; Lou, H.-X. Cancer Lett. 2008, 262, 173-182. doi:10.1016/j.canlet.2007.12.014

11. Shi, Y.-q.; Qu, X.-j.; Liao, Y.-x.; Xie, C.-f.; Cheng, Y.-n.; Li, S.; Lou, H.-x. Eur. J. Pharmacol. 2008, 584, 66-71. doi:10.1016/j.ejphar.2008.01.039

12. Asakawa, Y.; Ludwiczuk, A.; Nagashima, F.; Toyota, M.; Hashimoto, T.; Tori, M.; Fukuyama, Y.; Harinantenaina, L. Heterocycles 2009, 77, 99-150. doi:10.3987/REV-08-SR(F)3

13. Bringmann, G.; Mühlbacher, J.; Reichert, M.; Dreyer, M.; Kolz, J.; Speicher, A. J. Am. Chem. Soc. 2004, 126, 9283-9290. doi:10.1021/ja0373162

14. Scher, J. M.; Zapp, J.; Becker, H.; Kather, N.; Kolz, J.; Speicher, A.; Dreyer, M.; Maksimenka, K.; Bringmann, G. Tetrahedron 2004, 60, 9877-9881. doi:10.1016/j.tet.2004.08.037

15. Eicher, T.; Fey, S.; Puhl, W.; Büchel, E.; Speicher, A. Eur. J. Org. Chem. 1998, 877-888. doi:10.1002/(SICI)1099-0690(199805)1998:5<877::AID-EJOC877>3.0 CO;2-S

16. Hesse, M.; Meier, H.; Zeeh, B. Spectroscopic Methods in Organic Chemistry, 2nd ed.; Thieme Chemistry: Stuttgart, Germany, 2007.

17. Jackmann, L. M.; Fernandes, E.; Heubes, M.; Quast, H. Eur. J. Org. Chem. 1998, 2209-2227. doi:10.1002/(SICI)1099-0690(199810)1998:10<2209::AID-EJOC2209> 3.0.CO;2-G

18. HyperChem Professional 7.52; Hypercube, Inc.: Gainesville, FL, USA, 2002.

19. Speicher, A.; Backes, T.; Grosse, S. Tetrahedron 2005, 61, 11692-11696. doi:10.1016/j.tet.2005.09.048

20. Speicher, A.; Kolz, J.; Sambanje, R. P. Synthesis 2002, 2503-2512. doi:10.1055/s-2002-35629

21. Pachaly, P.; Schäfer, M. Arch. Pharm. 1989, 322, 477-482. doi:10.1002/ardp.19893220805

22. Gopinath, R.; Haque, S. J.; Patel, B. K. J. Org. Chem. 2002, 67, 5842-5845. doi:10.1021/jo025701o

23. Matsumoto, M.; Kuroda, K. Tetrahedron Lett. 1980, 21, 4021-4024. doi:10.1016/S0040-4039(00)92860-0

24. Kuwabe, S.-i.; Torraca, K. E.; Buchwald, S. L. J. Am. Chem. Soc. 2001, 123, 12202-12206. doi:10.1021/ja012046d 
25. Elangovan, A.; Wang, Y.-H.; Ho, T.-I. Org. Lett. 2003, 5, 1841-1844. doi:10.1021/ol034320+

26. Ma, D.; Liu, F. Chem. Commun. 2004, 1934-1935. doi:10.1039/b407090a

27. Beak, P.; Musick, T. J.; Chen, C.-w. J. Am. Chem. Soc. 1988, 110, 3538-3542. doi:10.1021/ja00219a031

28. Mounetou, E.; Debiton, E.; Buchdahl, C.; Gardette, D.; Gramain, J.-C.; Maurizis, J.-C.; Veyre, A.; Madelmont, J.-C. J. Med. Chem. 1997, 40, 2902-2909. doi:10.1021/jm960881d

29. Lee, C. K.; Koo, B.-S.; Lee, Y. S.; Cho, H. K.; Lee, K.-J. Bull. Korean Chem. Soc. 2002, 23, 1667-1670.

30. Murata, M.; Oyama, T.; Watanabe, S.; Masuda, Y. J. Org. Chem. 2000, 65, 164-168. doi:10.1021/jo991337q

31. Cockerill, G. S.; Levett, P. C.; Whiting, D. A. J. Chem. Soc., Perkin Trans. 1 1995, 9, 1103-1113. doi:10.1039/p19950001103

32. McMurry, J.; Matz, J. R.; Kees, K. L.; Bock, P. A. Tetrahedron Lett. 1982, 23, 1777-1780. doi:10.1016/S0040-4039(00)86739-8

33. Ali, M. A.; Kondo, K.; Tsuda, Y. Chem. Pharm. Bull. 1992, 40, 1130-1136.

34. Solladié, G.; Pasturel-Jacopé, Y.; Maignan, J. Tetrahedron 2003, 59, 3315-3321. doi:10.1016/S0040-4020(03)00405-8

\section{License and Terms}

This is an Open Access article under the terms of the Creative Commons Attribution License

(http://creativecommons.org/licenses/by/2.0), which permits unrestricted use, distribution, and reproduction in any medium, provided the original work is properly cited.

The license is subject to the Beilstein Journal of Organic Chemistry terms and conditions:

(http://www.beilstein-journals.org/bjoc)

The definitive version of this article is the electronic one which can be found at: doi:10.3762/bjoc.5.71 